\title{
Prosthetic Management of Maxillary Defect Due to Mucormycosis - A Case Report
}

\section{Hemant Gadge ${ }^{1 *}$, Mahesh Gandhewar ${ }^{2}$, Sumanth Kori ${ }^{3}$, Suresh Nagaral $^{4}$, Nageshnath Waghmare ${ }^{5}$ and Ashwini Pungle ${ }^{5}$}

${ }^{1}$ Reader, Department of Prosthodontics, A.C.P.M Dental College, Dhule,

Maharashtra, India

${ }^{2}$ Professor and Head of Department, Department of Prosthodontics, A.C.P.M Dental

College, Dhule, Maharashtra, India

${ }^{3}$ Head of Dental Department, Alia International Hospital, Kuwait

4Professor, Department of Prosthodontics, A.C.P.M Dental College, Dhule,

Maharashtra, India

${ }^{5}$ Post graduate student, Department of Prosthodontics, A.C.P.M Dental College,

Dhule, Maharashtra, India

*Corresponding Author: Hemant Gadge, Reader, Department of Prosthodontics,

A.C.P.M Dental College, Dhule, Maharashtra, India.
Received: October 14, 2021

Published: November 30, 2021

(C) All rights are reserved by Hemant Gadge., et al.

\section{Abstract}

This clinical report describes management of old diabetic patient with mucormycosis by surgical debridement, systemic antifungal administration and prosthetic adjunctive therapy and using hollow obturator. Restoration of esthetic and cosmetic appearance of patient along with restoration of function, protection of tissues, therapeutic and healing effect, psychological therapy and improving the quality of life are main objectives of rehabilitation.

The rehabilitation successfully done with a hollow bulb obturator in a case presented with subtotal maxillectomy is explained in given case report.

Keywords: Hemi-maxillectomy; Mucormycosis; Hollow Obturator; Oro-antral Communication

\section{Introduction}

Resection of maxilla due to tumours or any infection leads to facial disfigurement, impaired functions like speech due to hypernasality, rumination, and a significant impact on patients quality of life. Post COVID-19 sepsis is that which occurs after SARS-CoV-2 had a rampage in the human body. It leads to secondary bacterial and fungal infections, especially in critically ill patients subjected to invasive emergency procedures, mechanical ventilation, prolonged hospital stays, and breaches in asepsis. Further, corticosteroid treatment in these highly susceptible hosts and high fungal spore counts in the environment creates the favourable condition for mold infections. Antifungal agents along with surgical debridement or resection are treatment modalities for such kind of infection. This article discusses an innovative fabrication technique of obturator to treat an acquired maxillary defect due to post Covid-19 mucormycosis [2].

Multidisciplinary approach is required for management of such maxillary defects treated with surgical resection. The most practical convenient and cost effective treatment mode along with advantage of removable prosthesis which helps in evaluation of infected side efficiently is provided by prosthodontic rehabilitation.

\section{Case Report}

Examination and treatment planning

A 60 years old male patient visited with chief complain of nasal regurgitation, inability to chew food with existing prosthesis and loss of appearance. 
On past medical history, it was found that the patient is diabetic since 4 years and underwent maxillectomy for post Covid-19 necrosis of maxilla due to mucormycosis six months back. The treatment plan was to fabricate a hollow bulb obturator.

On Extraoral examination facial asymmetry with collapsed cheek and prominent nasolabial fold on right side of the face was found.

On intraoral examination well healed residual maxillary defect with oro-antral communication due to partial maxillectomy on right side was found. On right side 11, 12, 13, 14, 15, 16, 17, 18 and on left side 21 teeth and alveolar ridge were missing with obliteration of labial and buccal vestibule on same side. On panaromic radigraphic examination we observed missing maxillary teeth on the right side with a radiolucency extending into the maxillary sinus.

After examining the defect, treatment plan was made. It was decided to rehabilitate the patient with hollow obturator after oral prophylaxis. On examining intraorally it was found that the defect present was of Aramany's Class-IV type. The retention to the obturator was obtained from Adams clasp and C clasps made on remaining dentition.

\section{Methods}

Initially, a primary impression was made with irreversible hydrocolloid impression material using a stock tray (Figure 1). After retrieval the primary cast, a special tray was fabricated with self-cure acrylic resin. Border moulding was done with greenstick material and the full extent of the defect was recorded using an impression compound. A zinc-oxide non-eugenol paste was used to make secondary impression after scraping $1 \mathrm{~mm}$ of impression compound to record the undercuts which aid in anatomic retention. A final pick-up impression was made with alginate to record existing dentate segment (Figure 2). The type IV gypsum was used to make master cast after beading and boxing of final impression (Figure 3 and 4). A C-clasp on lateral incisor as well as on $3^{\text {rd }}$ molar and Adams clasps on $1^{\text {st }}$ molar were given (Figure 5) after that block out (Figure 6) of master cast was done and record base of self cure acrylic was fabricated on master cast (Figure 7). On the record base occlusal rim was fabricated. Bite registration was done (Figure 8). After that maxillary and mandibular casts were mounted. Accordingly teeth were arranged (Figure 9). After that Try-in was done (Figure 10). Evaluation of Occlusion, aesthetics, and phonet- ics was done and found to be acceptable by the patient. A C-clasp on lateral incisor as well as on $3^{\text {rd }}$ molar and Adams clasps on $1^{\text {st }}$ molar were given. The final wax-up was done. For a obturator to make it hollow we used putty elastomeric material in defect area during packing. On obturator insertion necessary corrections and occlusal adjustments were made. After final finishing and polishing, insertion of the prosthesis was done (Figure 11 and 12) and post-insertion instructions were given. Results were satisfied after 1 month follow up. Definative treatment has been planned for the missing dentate segment of patient.

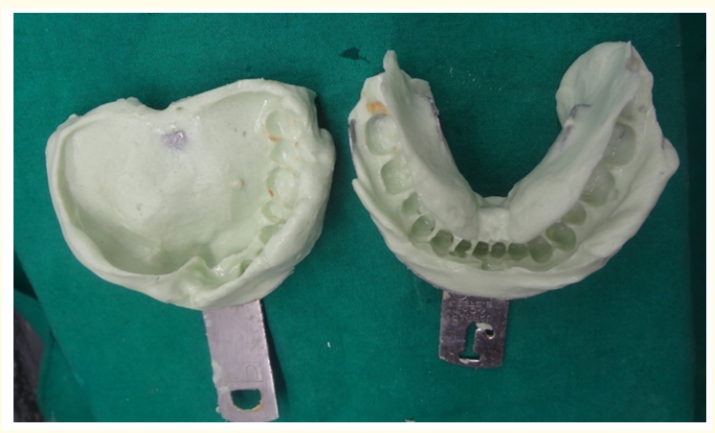

Figure 1: Primary Impression.

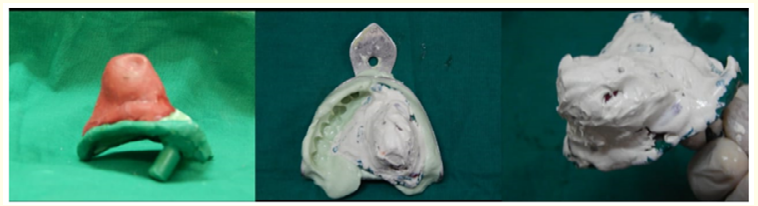

Figure 2: Final impression and Dual impression.

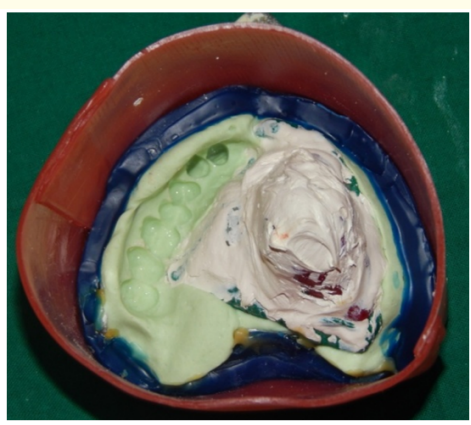

Figure 3: Beading and boxing of Final Impression. 


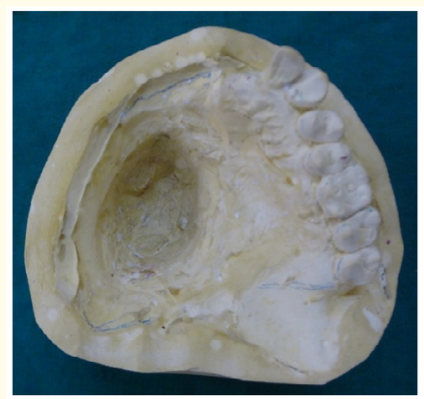

Figure 4: Master cast.

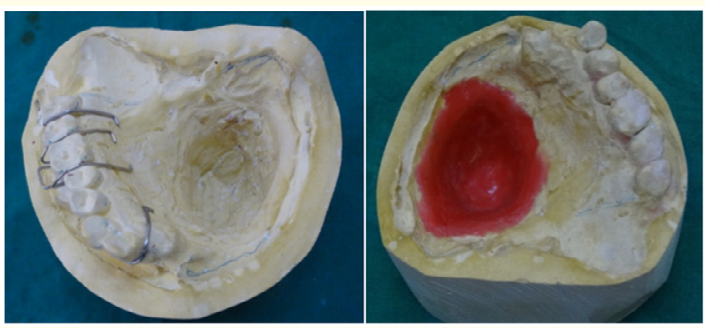

Figure 5: Master cast clasp.

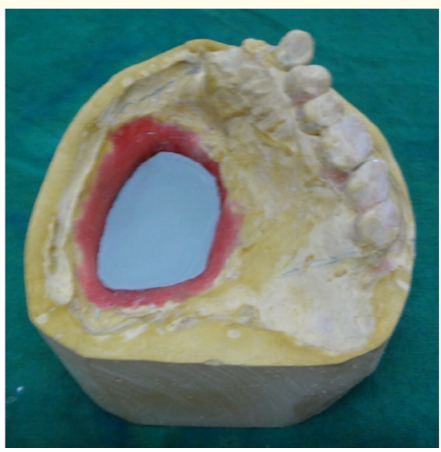

Figure 6: Master cast blockout.
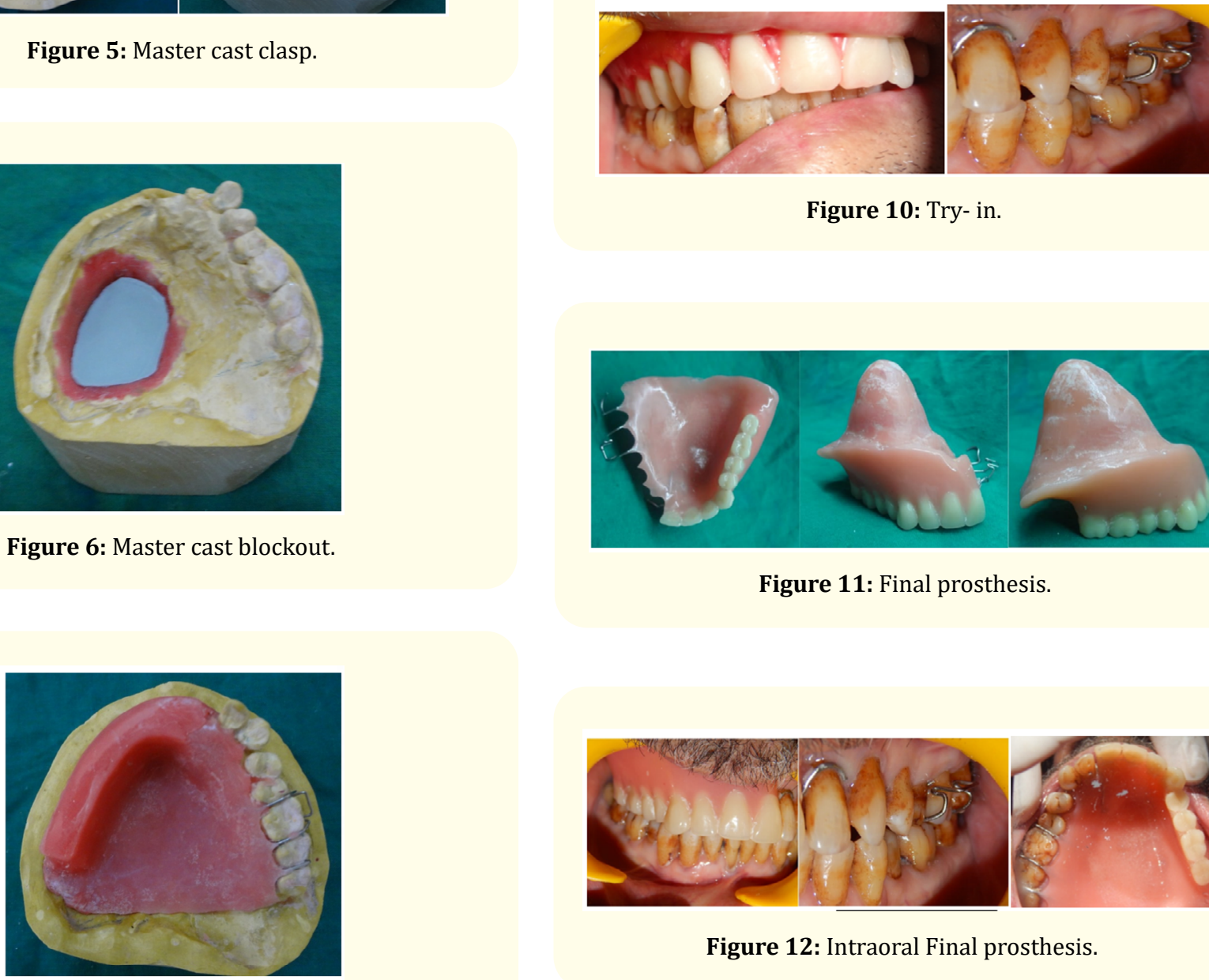

Figure 10: Try- in.

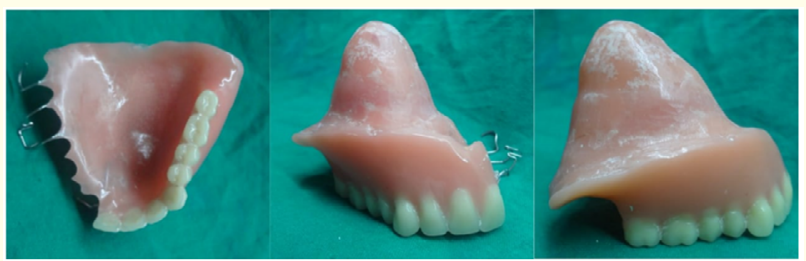

Figure 11: Final prosthesis.

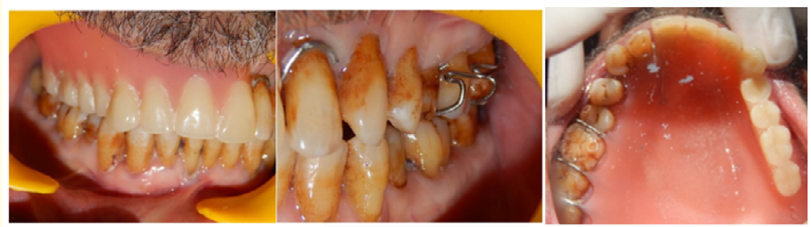

Figure 12: Intraoral Final prosthesis.

Figure 7: Record base and wax rim. 


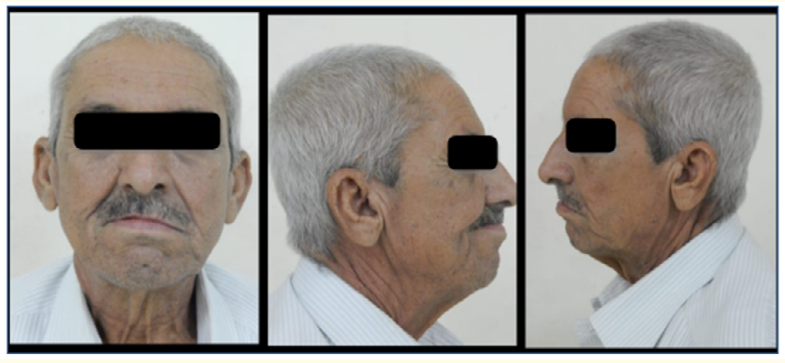

Figure 13: Preoperative view.

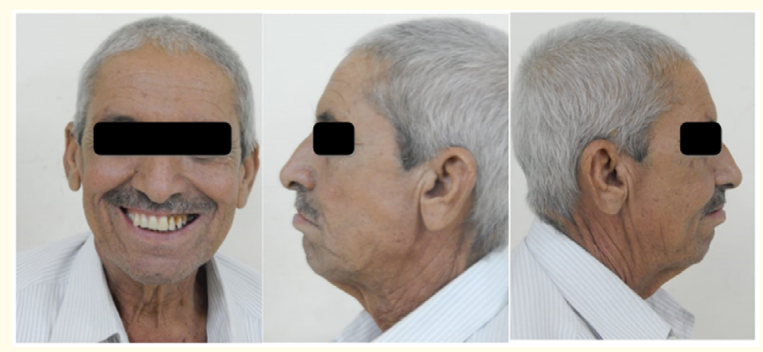

Figure 14: Post operative view.

\section{Discussion}

Mucormycosis is rare opportunistic fungal infection. According to the Jain., et al. surgical and anti-fungal treatment can be used in combination. Following are the types of mucormycosis - rhinocerebral, rhinomaxillary, pulmonary, cutaneous, gastrointestinal, and central nervous system. Among these rhinocerebral and pulmonary forms are predominant in diabetes patients. Rhinocerebral form occurs by inhalation of air borne spores. The action of mononuclear and poly morphonuclear phagocytes in the subcutaneous tissue begins immediately after inhalation of pores. As in diabetic patients, rhizopus produces ketoreductase an enzyme. That enzyme makes use of the patients ketone bodies leading to reduced phagocytic activity. Early diagnosis and early management prevent complications. Mucormycosis can be fatal to the patients. IV liposomal amphotericin, IV lipid complex, hyperbaric oxygen therapy are introduced recently. According to the extension of the obturator into the defect it can be classified as solid, open hollow, and closed hollow. Hollowing of the obturator makes it lightweight prosthesis whether it is open or closed hollow obturator that can be better accepted by the patient [3].
Maxillofacial rehabilitation is a multidisciplinary task. Communication with the surgeon as far as extent of disease, precise surgical technique, anticipated postoperative defects and healing time could help to plan the treatment. It is well established, among those involved in maxillofacial rehabilitation, that the fewer the teeth remaining in the arch, the more complicated is the closure of the maxillary defects by means of flaps. In particular, the separation between the oral and nasal cavity with a muscular diaphragm which does not rest on hard tissue leads to a mobile support, for which it is very difficult to achieve adequate impressions. Moreover, the support thus provided to the prosthesis gives way during mastication loads. Recommendations can be made for the preservation of tissues or to improve the existing anatomical structures to improve the retention, stability and support for the prosthesis. Coordination with speech pathologist to gain knowledge about mechanics and physiology of speech can help to design the prosthesis, which can fulfill the requirements of resonance, phonation and articulation. In the above presented cases, our aim was to eliminate the communication of the oral and nasal cavity by way of an obturator prosthesis, providing adequate functions of chewing, swallowing, and speech, as well as acceptable esthetic appearance [4].

The prosthetic rehabilitation of large maxillectomy defect is challenging task. Retention is severely compromised in these patients resulting in difficulties in speech and mastication [5].

Simple technique has been described in this article is for the fabrication of the hollow bulb obturator with clasps engaging the abutment teeth offer additional prosthetic retention which restores patient's original dentition and facial and palatal tissue form.

In the present case, additional retention was achieved by engaging clasps on the abutment teeth, circumferential clasps were given on opposite lateral incisor and $3^{\text {rd }}$ molar; and adams clasp on $1^{\text {st }}$ molar.

To establish occlusal function missing teeth was replaced with acrylic teeth.

Obturators with teeth may be made using several methods, using a celluloid matrix, modifying a surgical obturator, using a denture duplicator, or using light cured or heat-polymerized acrylic resin [6].

The obturator fabricated with this technique utilises heat cured acrylic denture base resin which is easily available in market. 
In this technique, putty elastomeric impression material is used during processing for fabrication of the hollow obturator, which is later removed by drilling a hole in the superior surface and the hole is filled with auto polymerizing resin. And trial denture was flasked, dewaxed and processed in customised flasks as conventional flask were not of adequate height.

The advantages of this hollow obturator are

- This prosthesis improves the esthetic of patient

- $\quad$ The use wire clasp in the prosthesis has improved the retention and enhanced the bracing effect

- Making the obturator hollow has reduced the weight of the prosthesis

- The use of the hollow obturator has improved masticatory function as well as the phonetics of the patient.

- The fabrication of this obturator is not technique sensitive it simple to fabricate

- $\quad$ Patient can easily insert the prosthesis intra-orally and can also maintain the hygiene properly.

- The hollow obturator is noninvasive

- The material we have used in fabrication of the prosthesis is biocompatible thus it does not irritate the tissue.

\section{Conclusion}

- Definitive prosthodontic treatment is one of the final therapies which are instituted and it attempts to alleviate any anatomical and functional deficiencies.

- A good prosthesis serves the purpose of rehabilitation of the patient's lost function and aesthetics also it gives confidence and comfort in society.

- The comprehensive technique described in this clinical report can be employed in any similar case. In an attempt to improve the retentive and stabilizing characteristics of an obturative prosthesis, an outline of the technical construction is proposed which makes the appliance light weight by creating a hollow inner chamber. The potential benefits of the technique are availability and ease of fabrication of the prosthesis with additional retention using clasps.

- The defect was closed, and a satisfactory esthetics, phonation and function was established.

\section{Bibliography}

1. Ferro KJ., et al. "The glossary of prosthodontic terms". Journal of Prosthetic Dentistry 17.5 (2017): e1-e105.

2. Gayathri Bandari D., et al. "Prosthetic Rehabilitation of A PostCovid Mucormycosis Maxillectomy Defect Using A Fused TwoPiece Hollow Obturator: A Fabrication Technique". European Journal of Molecular and Clinical Medicine 7.11 (2021): 85648569.

3. Naveen S., et al. "Mucormycosis of the Palate and its PostSurgical Management: A Case Report". Journal of International Oral Health 7.12 (2015): 134.

4. Shah RJ., et al. "Prosthetic rehabilitation of acquired maxillary defects secondary to mucormycosis: clinical cases". The Journal of Contemporary Dental Practice 15.2 (2014): 242-249.

5. Sridevi JR., et al. "Techniques for fabricating hollow obturator: two case reports". SRM Journal of Research in Dental Sciences 5.2 (2014): 143.

6. Shambharkar VI., et al. "A simple technique to fabricate a surgical obturator restoring the defect in original anatomical form". The Journal of Advanced Prosthodontics 3.2 (2011): 106-109.

7. Nesappan T and Ganapathy DM. "Prosthodontic Rehabilitation of Maxillary Defect in a Patient with Mucormycosis".

\section{Volume 5 Issue 12 December 2021}

(C) All rights are reserved by Hemant Gadge., et al. 